\title{
Florida Honey Bee Plants ${ }^{1}$
}

\section{Mary Christine Bammer, William H Kern, and Jamie D. Ellis ${ }^{2}$}

Several factors influence the flora throughout Florida, including annual freezes, average temperature, annual rainfall, and soil composition. Because of these variations, plants that grow well in one region may not grow well in another. Climate, plant communities, and timing of floral resources differ significantly between the three main regions in Florida: north Florida, central Florida, and south Florida (north Florida encompasses the panhandle region south through Alachua, Levy, Putnam, and Flagler counties. Central Florida includes Marion County south through Sarasota County. South Florida encompasses the remaining counties including the Keys) (Figure 1).

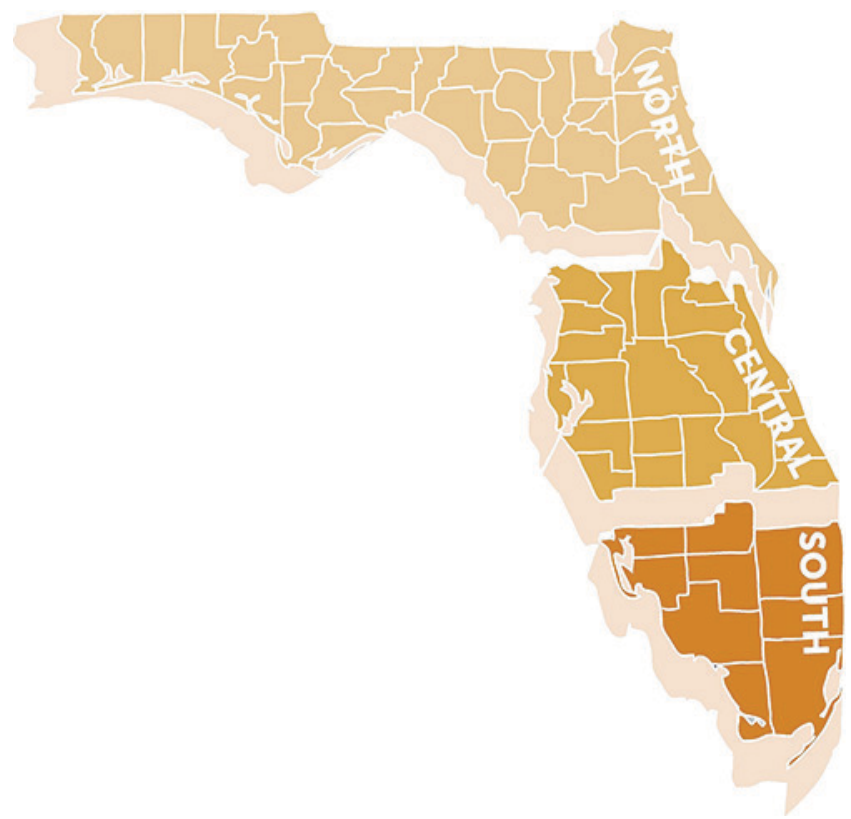

While many plants are acceptable pollen producers for honey bees, fewer yield enough nectar to produce a surplus honey crop. The tables in this document list the nectarbearing plants that are present to some degree in each region and the plants' respective bloom times. Please note, any nectar plants that are considered invasive in Florida have been excluded from this list.

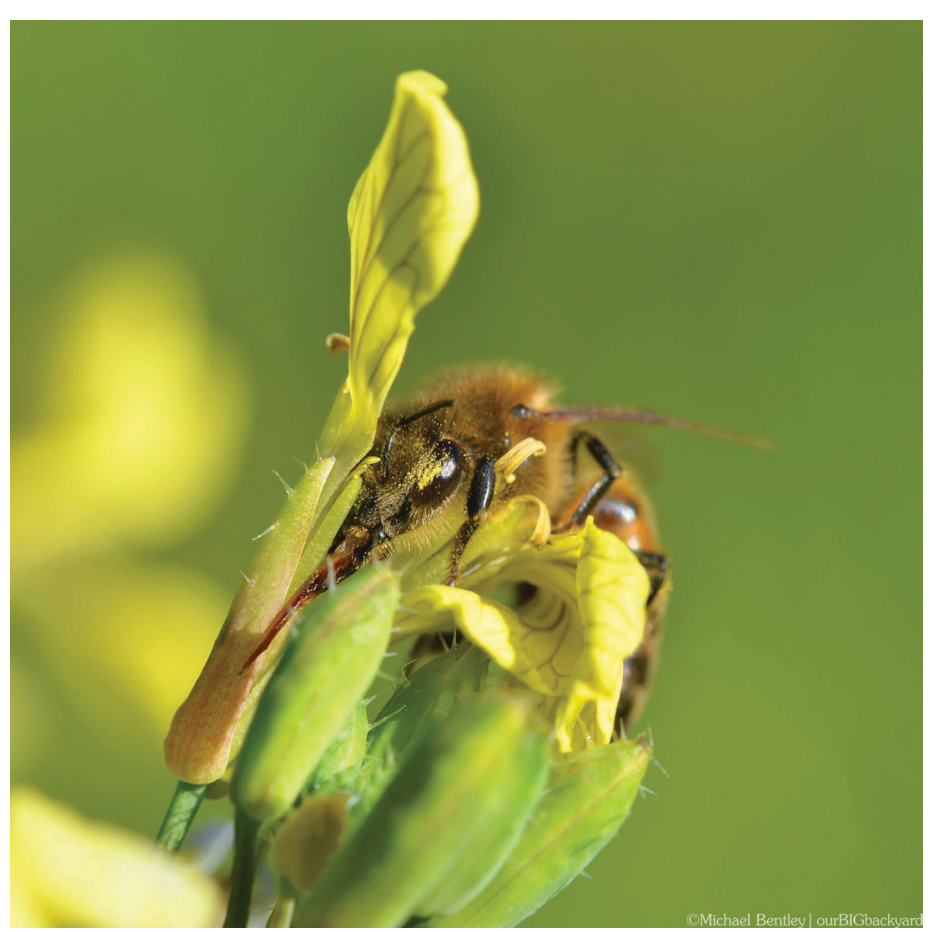

Figure 2. Honey bee on wild mustard.

Figure 1.

1. This document is ENY-171, one of a series of the Entomology and Nematology Department, UF/IFAS Extension. Original publication date September 2018. Visit the EDIS website at http://edis.ifas.ufl.edu.

2. Mary Christine Bammer, Extension coordinator, Department of Entomology and Nematology; William H Kern, associate professor of urban entomology, Department of Entomology and Nematology, UF/IFAS Ft. Lauderdale Research \& Education Center; and Jamie D. Ellis, associate professor, Department of Entomology and Nematology, UF/IFAS Extension, Gainesville, FL 32611.

The Institute of Food and Agricultural Sciences (IFAS) is an Equal Opportunity Institution authorized to provide research, educational information and other services

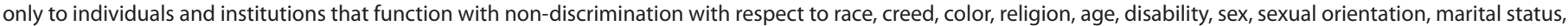
national origin, political opinions or affiliations. For more information on obtaining other UF/IFAS Extension publications, contact your county's UF/IFAS Extension office. U.S. Department of Agriculture, UF/IFAS Extension Service, University of Florida, IFAS, Florida A \& M University Cooperative Extension Program, and Boards of County Commissioners Cooperating. Nick T. Place, dean for UF/IFAS Extension. 
Table 1. Honey bee plant bloom calendar for North Florida.

\begin{tabular}{|c|c|c|}
\hline Month & Common Name & Scientific Name \\
\hline \multirow[t]{4}{*}{ January } & Maple & Acer spp. \\
\hline & Sand Pine & Pinus clausa \\
\hline & Wild Mustard & Sinapis arvensis \\
\hline & Willow & Salix spp. \\
\hline \multirow[t]{13}{*}{ February } & Blueberry & Vaccinium spp. \\
\hline & Cherry & Prunus spp. \\
\hline & Fetterbush & Lyonia lucida \\
\hline & Hawthorn & Crataegus spp. \\
\hline & Maple & Acer spp. \\
\hline & Oak & Quercus spp. \\
\hline & Plum & Prunus spp. \\
\hline & Spanish Needle & Bidens spp. \\
\hline & Sparkleberry & Vaccinium arboreum \\
\hline & Spring Titi, Buckwheat tree & Cliftonia monophylla \\
\hline & Sweet Clover & Melilotus officinalis \\
\hline & Walter Viburnum & Viburnum obovatum \\
\hline & Willow & Salix spp. \\
\hline \multirow[t]{13}{*}{ March } & Blueberry & Vaccinium spp. \\
\hline & Cherry & Prunus spp. \\
\hline & Fetterbush & Lyonia lucida \\
\hline & Hawthorn & Crataegus spp. \\
\hline & Oak & Quercus spp. \\
\hline & Orange & Poncirus trifoliata, Citrus reticulata \\
\hline & Plum & Prunus spp. \\
\hline & Spanish Needle & Bidens spp. \\
\hline & Sparkleberry & Vaccinium arboreum \\
\hline & Spring Titi, Buckwheat tree & Cliftonia monophylla \\
\hline & Sweet Clover & Melilotus officinalis \\
\hline & Walter Viburnum & Viburnum obovatum \\
\hline & Willow & Salix spp. \\
\hline \multirow[t]{15}{*}{ April } & American Holly & Ilex ораса \\
\hline & Black (Swamp) Tupelo, Blackgum & Nyssa sylvatica \\
\hline & Blackberry & Rubus spp. \\
\hline & Butter Mint, Buttermint, bushmints & Hyptis spp. \\
\hline & Dog Hobble & Leucothoe spp. \\
\hline & Fetterbush & Lyonia lucida \\
\hline & Galberry & Ilex glabra \\
\hline & Hawthorn & Crataegus spp. \\
\hline & Mexican Clover & Richardia spp. \\
\hline & Ogeechee (White) Tupelo & Nyssa ogeche \\
\hline & Orange & Poncirus trifoliata, Citrus reticulata \\
\hline & Palmetto, Cabbage Palm & Sabal palmetto \\
\hline & Spanish Needle & Bidens spp. \\
\hline & Sparkleberry & Vaccinium arboreum \\
\hline & Swamp Galberry & Ilex coriacea \\
\hline
\end{tabular}




\begin{tabular}{|c|c|c|}
\hline Month & Common Name & Scientific Name \\
\hline \multirow[t]{6}{*}{ April cont. } & Swamp Titi & Cyrilla racemiflora \\
\hline & Sweet Clover & Melilotus officinalis \\
\hline & Tuliptree & Liriodendron tulipifera \\
\hline & Water Locust & Gleditsia aquatica \\
\hline & Water Tupelo & Nyssa aquatica \\
\hline & Yaupon Holly & Ilex vomitoria \\
\hline \multirow[t]{28}{*}{ May } & American Holly & Ilex opaca \\
\hline & Black (Swamp) Tupelo, Blackgum & Nyssa sylvatica \\
\hline & Blackberry & Rubus spp. \\
\hline & Blackhaw & Viburnum prunifolium \\
\hline & Butter Mint, Buttermint, bushmints & Hyptis spp. \\
\hline & Cabbage Palm & Sabal palmetto \\
\hline & Chinese Privit & Ligustrum sinense \\
\hline & Dahoon Holly & Ilex cassine \\
\hline & Dog Hobble & Leucothoe spp. \\
\hline & Fetterbush & Lyonia lucida \\
\hline & Galberry & Ilex glabra \\
\hline & Gopher Apple & Licania michauxii \\
\hline & Joint Weed & Polygonella spp. \\
\hline & Mexican Clover & Richardia spp. \\
\hline & Ogeechee (White) Tupelo & Nyssa ogeche \\
\hline & Partridge Pea & Chamaecrista fasciculata \\
\hline & Possum Haw & Ilex decidua \\
\hline & Red Bay & Persea borbonia \\
\hline & Sandhill Prarie Clover & Dalea spp. \\
\hline & Saw Palmetto & Serenoa repens \\
\hline & Spanish Needle & Bidens spp. \\
\hline & Sparkleberry & Vaccinium arboreum \\
\hline & Spiderwort/Dayflower & Tradescantia virginiana \\
\hline & Swamp Bay & Persea palustris \\
\hline & Swamp Titi & Cyrilla racemiflora \\
\hline & Tuliptree & Liriodendron tulipifera \\
\hline & Water Tupelo & Nyssa aquatica \\
\hline & Yaupon Holly & Ilex vomitoria \\
\hline \multirow[t]{11}{*}{ June } & Black Mangrove & Avicennia germinans \\
\hline & Butter Mint, Buttermint, bushmints & Hyptis spp. \\
\hline & Chinese Privit & Ligustrum sinense \\
\hline & Chinese Tallow & Triadica sebifera \\
\hline & Dahoon Holly & Ilex cassine \\
\hline & Dog Hobble & Leucothoe spp. \\
\hline & Elderberry & Sambucus canadensis \\
\hline & Galberry & Ilex glabra \\
\hline & Gopher Apple & Licania michauxii \\
\hline & Joint Weed & Polygonella spp. \\
\hline & Loblolly Bay & Gordonia lasianthus \\
\hline
\end{tabular}




\begin{tabular}{|c|c|c|}
\hline Month & Common Name & Scientific Name \\
\hline \multirow[t]{13}{*}{ June cont. } & Mexican Clover & Richardia spp. \\
\hline & Palmetto, Cabbage Palm & Sabal palmetto \\
\hline & Partridge Pea & Chamaecrista fasciculata \\
\hline & Possum Haw & Ilex decidua \\
\hline & Red Bay & Persea borbonia \\
\hline & Red Cabbage Palm & Livistona mariae \\
\hline & Red Mangrove & Rhizophora mangle \\
\hline & Sandhill Prarie Clover & Dalea spp. \\
\hline & Spanish Needle & Bidens spp. \\
\hline & Spiderwort/Dayflower & Tradescantia virginiana \\
\hline & Swamp Bay & Persea palustris \\
\hline & Swamp Titi & Cyrilla racemiflora \\
\hline & White Mangrove & Laguncularia racemosa \\
\hline \multirow[t]{19}{*}{ July } & Black Mangrove & Avicennia germinans \\
\hline & Butter Mint, Buttermint, bushmints & Hyptis spp. \\
\hline & Cotton & Gossypium spp. \\
\hline & Elderberry & Sambucus canadensis \\
\hline & Gopher Apple & Licania michauxii \\
\hline & Joint Weed & Polygonella spp. \\
\hline & Loblolly Bay & Gordonia lasianthus \\
\hline & Mexican Clover & Richardia spp. \\
\hline & Palmetto, Cabbage Palm & Sabal palmetto \\
\hline & Partridge Pea & Chamaecrista fasciculata \\
\hline & Primrose Willow & Ludwigia spp. \\
\hline & Red Bay & Persea borbonia \\
\hline & Red Mangrove & Rhizophora mangle \\
\hline & Sandhill Prarie Clover & Dalea spp. \\
\hline & Saw Palmetto & Serenoa repens \\
\hline & Spanish Needle & Bidens spp. \\
\hline & Spiderwort/Dayflower & Tradescantia virginiana \\
\hline & Swamp Titi & Cyrilla racemiflora \\
\hline & White Mangrove & Laguncularia racemosa \\
\hline \multirow[t]{14}{*}{ August } & Black Mangrove & Avicennia germinans \\
\hline & Cotton & Gossypium spp. \\
\hline & Goldenrod & Solidago spp. \\
\hline & Joint Weed & Polygonella spp. \\
\hline & Loblolly Bay & Gordonia lasianthus \\
\hline & Mexican Clover & Richardia spp. \\
\hline & Partridge Pea & Chamaecrista fasciculata \\
\hline & Primrose Willow & Ludwigia spp. \\
\hline & Red Bay & Persea borbonia \\
\hline & Red Mangrove & Rhizophora mangle \\
\hline & Sandhill Prarie Clover & Dalea spp. \\
\hline & Spanish Needle & Bidens spp. \\
\hline & Spiderwort/Dayflower & Tradescantia virginiana \\
\hline & Spotted Mint & Monarda punctata \\
\hline
\end{tabular}




\begin{tabular}{|c|c|c|}
\hline Month & Common Name & Scientific Name \\
\hline \multirow[t]{3}{*}{ August cont. } & Sumac & Rhus typhina \\
\hline & Vine Aster & Ampelaster carolinianus \\
\hline & White Mangrove & Laguncularia racemosa \\
\hline \multirow[t]{11}{*}{ September } & Bush Aster & Aster spp. \\
\hline & Goldenrod & Solidago spp. \\
\hline & Mexican Clover & Richardia spp. \\
\hline & Primrose Willow & Ludwigia spp. \\
\hline & Red Bay & Persea borbonia \\
\hline & Smart Weed & Polygonum spp. \\
\hline & Spanish Needle & Bidens spp. \\
\hline & Spiderwort/Dayflower & Tradescantia virginiana \\
\hline & Spotted Mint & Monarda punctata \\
\hline & Sumac & Rhus typhina \\
\hline & Vine Aster & Ampelaster carolinianus \\
\hline \multirow[t]{9}{*}{ October } & Bush Aster & Aster spp. \\
\hline & Goldenrod & Solidago spp. \\
\hline & Mexican Clover & Richardia spp. \\
\hline & Primrose Willow & Ludwigia spp. \\
\hline & Smart Weed & Polygonum spp. \\
\hline & Spanish Needle & Bidens spp. \\
\hline & Spotted Mint & Monarda punctata \\
\hline & Vine Aster & Ampelaster carolinianus \\
\hline & Wild Mustard & Sinapis arvensis \\
\hline \multirow[t]{9}{*}{ November } & Bush Aster & Aster spp. \\
\hline & Goldenrod & Solidago spp. \\
\hline & Mexican Clover & Richardia spp. \\
\hline & Primrose Willow & Ludwigia spp. \\
\hline & Smart Weed & Polygonum spp. \\
\hline & Spanish Needle & Bidens spp. \\
\hline & Spotted Mint & Monarda punctata \\
\hline & Vine Aster & Ampelaster carolinianus \\
\hline & Wild Mustard & Sinapis arvensis \\
\hline \multirow[t]{5}{*}{ December } & Bush Aster & Aster spp. \\
\hline & Loquat & Eriobotrya japonica \\
\hline & Mist Flower & Conoclinium coelestinum \\
\hline & Spanish Needle & Bidens spp. \\
\hline & Wild Mustard & Sinapis arvensis \\
\hline
\end{tabular}


Table 2. Honey bee plant bloom calendar for Central Florida.

\begin{tabular}{|c|c|c|}
\hline Month & Common Name & Scientific Name \\
\hline \multirow[t]{4}{*}{ January } & Maple & Acer spp. \\
\hline & Sand Pine & Pinus clausa \\
\hline & Wild Mustard & Sinapis arvensis \\
\hline & Willow & Salix spp. \\
\hline \multirow[t]{12}{*}{ February } & Blueberry & Vaccinium spp. \\
\hline & Cherry & Prunus spp. \\
\hline & Fetterbush & Lyonia lucida \\
\hline & Hawthorn & Crataegus spp. \\
\hline & Maple & Acer spp. \\
\hline & Oak & Quercus spp. \\
\hline & Plum & Prunus spp. \\
\hline & Sand Pine & Pinus clausa \\
\hline & Swamp Titi & Cyrilla racemiflora \\
\hline & Sweet Clover & Melilotus officinalis \\
\hline & Walter Viburnum & Viburnum obovatum \\
\hline & Willow & Salix spp. \\
\hline \multirow[t]{11}{*}{ March } & Blueberry & Vaccinium spp. \\
\hline & Cherry & Prunus spp. \\
\hline & Fetterbush & Lyonia lucida \\
\hline & Hawthorn & Crataegus spp. \\
\hline & Oak & Quercus spp. \\
\hline & Orange & Poncirus trifoliata, Citrus reticulata \\
\hline & Plum & Prunus spp. \\
\hline & Spanish Needle & Bidens spp. \\
\hline & Sweet Clover & Melilotus officinalis \\
\hline & Walter Viburnum & Viburnum obovatum \\
\hline & Willow & Salix spp. \\
\hline \multirow[t]{15}{*}{ April } & American Beautyberry & Callicarpa americana \\
\hline & American Holly & Ilex opaca \\
\hline & Black (Swamp) Tupelo, Blackgum & Nyssa sylvatica \\
\hline & Blueberry & Vaccinium spp. \\
\hline & Butter Mint, Buttermint, bushmints & Hyptis spp. \\
\hline & Dog Hobble & Leucothoe spp. \\
\hline & Fetterbush & Lyonia lucida \\
\hline & Galberry & Ilex glabra \\
\hline & Hawthorn & Crataegus spp. \\
\hline & Mexican Clover & Richardia spp. \\
\hline & Orange & Poncirus trifoliata, Citrus reticulata \\
\hline & Palmetto, Cabbage Palm & Sabal palmetto \\
\hline & Spanish Needle & Bidens spp. \\
\hline & Sweet Clover & Melilotus officinalis \\
\hline & Yaupon Holly & Ilex vomitoria \\
\hline
\end{tabular}




\begin{tabular}{|c|c|c|}
\hline Month & Common Name & Scientific Name \\
\hline \multirow[t]{15}{*}{ May } & American Holly & Ilex opaca \\
\hline & Butter Mint, Buttermint, bushmints & Hyptis spp. \\
\hline & Dahoon Holly & Ilex cassine \\
\hline & Dog Hobble & Leucothoe spp. \\
\hline & Fetterbush & Lyonia lucida \\
\hline & Galberry & Ilex glabra \\
\hline & Gopher Apple & Licania michauxii \\
\hline & Joint Weed & Polygonella spp. \\
\hline & Mexican Clover & Richardia spp. \\
\hline & Ogeechee (White) Tupelo & Nyssa ogeche \\
\hline & Palm & many species \\
\hline & Palmetto, Cabbage Palm & Sabal palmetto \\
\hline & Sandhill Prarie Clover & Dalea spp. \\
\hline & Spiderwort/Dayflower & Tradescantia virginiana \\
\hline & Yaupon Holly & Ilex vomitoria \\
\hline \multirow[t]{19}{*}{ June } & Black Mangrove & Avicennia germinans \\
\hline & Butter Mint, Buttermint, bushmints & Hyptis spp. \\
\hline & Dahoon Holly & Ilex cassine \\
\hline & Dog Hobble & Leucothoe spp. \\
\hline & Elderberry & Sambucus canadensis \\
\hline & Gopher Apple & Licania michauxii \\
\hline & Joint Weed & Polygonella spp. \\
\hline & Loblolly Bay & Gordonia lasianthus \\
\hline & Mexican Clover & Richardia spp. \\
\hline & Palm & many species \\
\hline & Palmetto, Cabbage Palm & Sabal palmetto \\
\hline & Red Bay & Persea borbonia \\
\hline & Red Mangrove & Rhizophora mangle \\
\hline & Sandhill Prarie Clover & Dalea spp. \\
\hline & Spanish Needle & Bidens spp. \\
\hline & Spiderwort/Dayflower & Tradescantia virginiana \\
\hline & Virginia Creeper & Parthenocissus quinquefolia \\
\hline & Walter Viburnum & Viburnum obovatum \\
\hline & White Mangrove & Laguncularia racemosa \\
\hline \multirow[t]{11}{*}{ July } & Black Mangrove & Avicennia germinans \\
\hline & Butter Mint, Buttermint, bushmints & Hyptis spp. \\
\hline & Elderberry & Sambucus canadensis \\
\hline & Gopher Apple & Licania michauxii \\
\hline & Joint Weed & Polygonella spp. \\
\hline & Loblolly Bay & Gordonia lasianthus \\
\hline & Mexican Clover & Richardia spp. \\
\hline & Palm & many species \\
\hline & Palmetto, Cabbage Palm & Sabal palmetto \\
\hline & Partridge Pea & Chamaecrista fasciculata \\
\hline & Primrose Willow & Ludwigia spp. \\
\hline
\end{tabular}




\begin{tabular}{|c|c|c|}
\hline Month & Common Name & Scientific Name \\
\hline \multirow[t]{6}{*}{ July cont. } & Red Bay & Persea borbonia \\
\hline & Red Mangrove & Rhizophora mangle \\
\hline & Sandhill Prarie Clover & Dalea spp. \\
\hline & Spanish Needle & Bidens spp. \\
\hline & Spiderwort/Dayflower & Tradescantia virginiana \\
\hline & White Mangrove & Laguncularia racemosa \\
\hline \multirow[t]{15}{*}{ August } & Black Mangrove & Avicennia germinans \\
\hline & Goldenrod & Solidago spp. \\
\hline & Joint Weed & Polygonella spp. \\
\hline & Mexican Clover & Richardia spp. \\
\hline & Partridge Pea & Chamaecrista fasciculata \\
\hline & Primrose Willow & Ludwigia spp. \\
\hline & Red Bay & Persea borbonia \\
\hline & Red Mangrove & Rhizophora mangle \\
\hline & Sandhill Prarie Clover & Dalea spp. \\
\hline & Spanish Needle & Bidens spp. \\
\hline & Spiderwort/Dayflower & Tradescantia virginiana \\
\hline & Spotted Mint & Monarda punctata \\
\hline & Sumac & Rhus typhina \\
\hline & Vine Aster & Ampelaster carolinianus \\
\hline & White Mangrove & Laguncularia racemosa \\
\hline \multirow[t]{11}{*}{ September } & Bush Aster & Aster spp. \\
\hline & Goldenrod & Solidago spp. \\
\hline & Mexican Clover & Richardia spp. \\
\hline & Primrose Willow & Ludwigia spp. \\
\hline & Red Bay & Persea borbonia \\
\hline & Smart Weed & Polygonum spp. \\
\hline & Spanish Needle & Bidens spp. \\
\hline & Spiderwort/Dayflower & Tradescantia virginiana \\
\hline & Spotted Mint & Monarda punctata \\
\hline & Sumac & Rhus typhina \\
\hline & Vine Aster & Ampelaster carolinianus \\
\hline \multirow[t]{8}{*}{ October } & Bush Aster & Aster spp. \\
\hline & Goldenrod & Solidago spp. \\
\hline & Mexican Clover & Richardia spp. \\
\hline & Primrose Willow & Ludwigia spp. \\
\hline & Smart Weed & Polygonum spp. \\
\hline & Spanish Needle & Bidens spp. \\
\hline & Spotted Mint & Monarda punctata \\
\hline & Vine Aster & Ampelaster carolinianus \\
\hline
\end{tabular}




\begin{tabular}{|l|l|l|}
\hline \multicolumn{1}{|c|}{ Month } & \multicolumn{1}{c|}{ Common Name } & \multicolumn{1}{c|}{ Scientific Name } \\
\hline \multirow{5}{*}{ November } & Bush Aster & Aster spp. \\
\cline { 2 - 3 } & Goldenrod & Solidago spp. \\
\hline & Mexican Clover & Richardia spp. \\
\cline { 2 - 3 } & Primrose Willow & Ludwigia spp. \\
\hline & Smart Weed & Polygonum spp. \\
\hline & Spanish Needle & Bidens spp. \\
\hline & Spotted Mint & Monarda punctata \\
\hline \multirow{5}{*}{ December } & Vine Aster & Ampelaster carolinianus \\
\hline & Bush Aster & Aster spp. \\
\hline & Goldenrod & Solidago spp. \\
\hline & Mexican Clover & Richardia spp. \\
\hline & Primrose Willow & Ludwigia spp. \\
\hline & Spanish Needle & Bidens spp. \\
\hline & Wild Radish/Wild Mustard & Raphanus raphanistrum \\
\hline
\end{tabular}

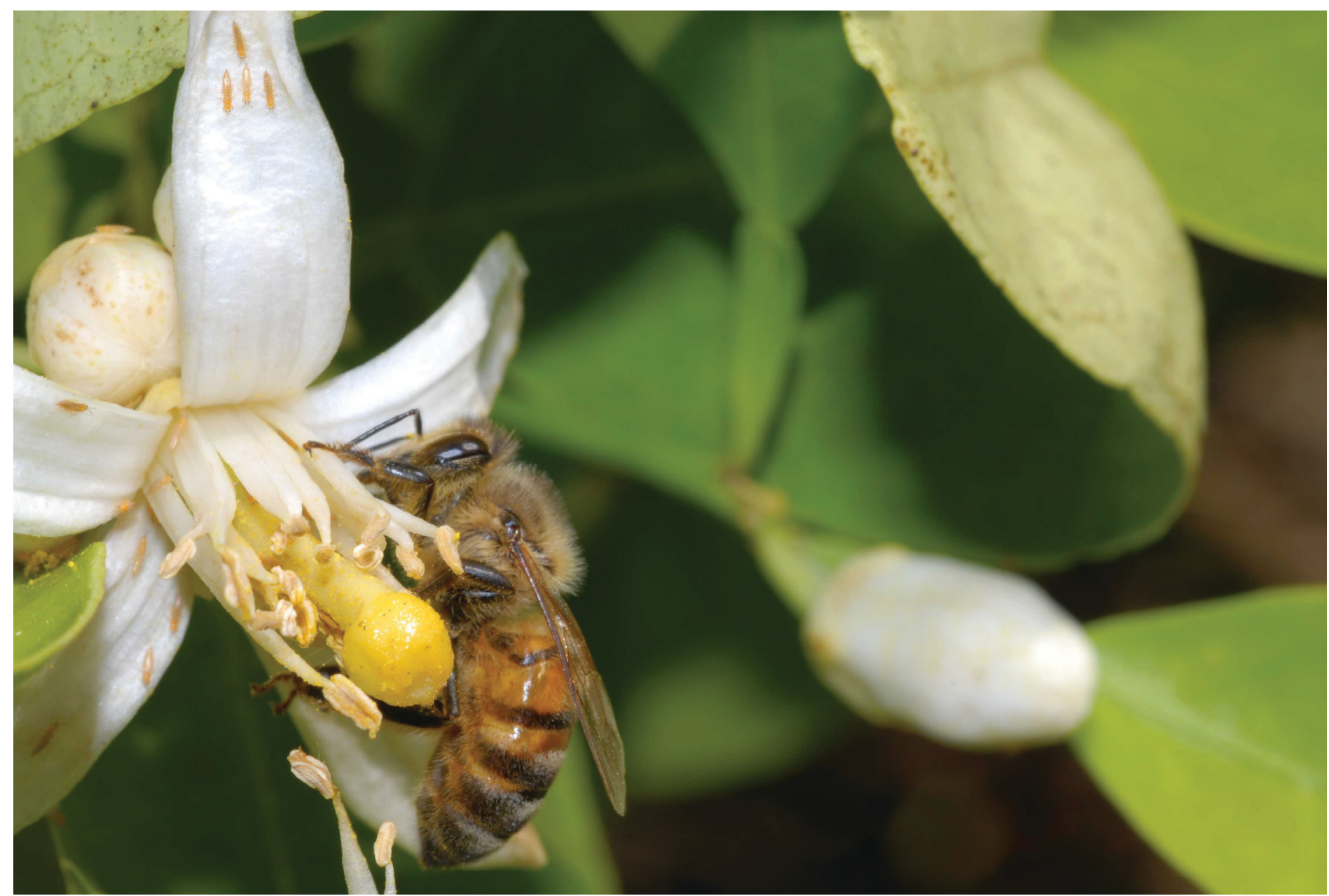

Figure 3. Honey bee on citrus. 
Table 3. Honey bee plant bloom calendar for South Florida.

\begin{tabular}{|c|c|c|}
\hline Month & Common Name & Scientific Name \\
\hline \multirow[t]{6}{*}{ January } & Maple & Acer spp. \\
\hline & Mexican Clover & Richardia spp. \\
\hline & Primrose Willow & Ludwigia spp. \\
\hline & Spanish Needle & Bidens spp. \\
\hline & Wild Mustard & Sinapis arvensis \\
\hline & Willow & Salix spp. \\
\hline \multirow[t]{10}{*}{ February } & American Beautyberry & Callicarpa americana \\
\hline & Blackberry & Rubus spp. \\
\hline & Coreopsis & Coreopsis spp. \\
\hline & Mexican Clover & Richardia spp. \\
\hline & Oak & Quercus spp. \\
\hline & Orange & Poncirus trifoliata, Citrus reticulata \\
\hline & Primrose Willow & Ludwigia spp. \\
\hline & Spanish Needle & Bidens spp. \\
\hline & Sweet Acacia & Vachellia farnesiana \\
\hline & Sweet Clover & Melilotus officinalis \\
\hline \multirow[t]{11}{*}{ March } & American Beautyberry & Callicarpa americana \\
\hline & Buttonwood & Conocarpus erectus \\
\hline & Lychee & Litchi chinensis \\
\hline & Mexican Clover & Richardia spp. \\
\hline & Oak & Quercus spp. \\
\hline & Orange & Poncirus trifoliata, Citrus reticulata \\
\hline & Primrose Willow & Ludwigia spp. \\
\hline & Saw Palmetto & Serenoa repens \\
\hline & Seagrape & Coccoloba uvifera \\
\hline & Spanish Needle & Bidens spp. \\
\hline & Sweet Clover & Melilotus officinalis \\
\hline \multirow[t]{16}{*}{ April } & American Beautyberry & Callicarpa americana \\
\hline & Button sage & Lantana involucrata \\
\hline & Buttonwood & Conocarpus erectus \\
\hline & Galberry & Ilex glabra \\
\hline & Macadamia & Macadamia spp. \\
\hline & Mexican Clover & Richardia spp. \\
\hline & Orange & Poncirus trifoliata, Citrus reticulata \\
\hline & Pineland lantana & Lantana depressa \\
\hline & Primrose Willow & Ludwigia spp. \\
\hline & Seagrape & Coccoloba uvifera \\
\hline & Shrubby False Buttonweed & Spermacoce verticillata \\
\hline & Smart Weed & Polygonum spp. \\
\hline & Spanish Needle & Bidens spp. \\
\hline & White Mangrove & Laguncularia racemosa \\
\hline & Wild Coffee & Psychotria nervosa \\
\hline & Wild lime & Zanthoxylum fagara \\
\hline
\end{tabular}




\begin{tabular}{|c|c|c|}
\hline Month & Common Name & Scientific Name \\
\hline \multirow[t]{12}{*}{ May } & Buttonwood & Conocarpus erectus \\
\hline & Dahoon Holly & Ilex cassine \\
\hline & Galberry & Ilex glabra \\
\hline & Mexican Clover & Richardia spp. \\
\hline & Orange & Poncirus trifoliata, Citrus reticulata \\
\hline & Palmetto, Cabbage Palm & Sabal palmetto \\
\hline & Primrose Willow & Ludwigia spp. \\
\hline & Seagrape & Coccoloba uvifera \\
\hline & Shrubby False Buttonweed & Spermacoce verticillata \\
\hline & Smart Weed & Polygonum spp. \\
\hline & Spanish Needle & Bidens spp. \\
\hline & Wild Coffee & Psychotria nervosa \\
\hline \multirow[t]{20}{*}{ June } & Black Mangrove & Avicennia germinans \\
\hline & Cashew & Anacardium spp. \\
\hline & Dahoon Holly & Ilex cassine \\
\hline & Elderberry & Sambucus canadensis \\
\hline & Fiddlewood & Citharexylum spinosum \\
\hline & Galberry & Ilex glabra \\
\hline & Mexican Clover & Richardia spp. \\
\hline & Palm & many species \\
\hline & Palmetto, Cabbage Palm & Sabal palmetto \\
\hline & Primrose Willow & Ludwigia spp. \\
\hline & Red Mangrove & Rhizophora mangle \\
\hline & Seagrape & Coccoloba uvifera \\
\hline & Shrubby False Buttonweed & Spermacoce verticillata \\
\hline & Smart Weed & Polygonum spp. \\
\hline & Spanish Needle & Bidens spp. \\
\hline & Sweet Acacia & Vachellia farnesiana \\
\hline & Virginia Creeper & Parthenocissus quinquefolia \\
\hline & Walter Viburnum & Viburnum obovatum \\
\hline & White Mangrove & Laguncularia racemosa \\
\hline & Wild Coffee & Psychotria nervosa \\
\hline \multirow[t]{12}{*}{ July } & Black Mangrove & Avicennia germinans \\
\hline & Elderberry & Sambucus canadensis \\
\hline & Mexican Clover & Richardia spp. \\
\hline & Palm & many species \\
\hline & Palmetto, Cabbage Palm & Sabal palmetto \\
\hline & Primrose Willow & Ludwigia spp. \\
\hline & Red Mangrove & Rhizophora mangle \\
\hline & Shrubby False Buttonweed & Spermacoce verticillata \\
\hline & Smart Weed & Polygonum spp. \\
\hline & Spanish Needle & Bidens spp. \\
\hline & White Mangrove & Laguncularia racemosa \\
\hline & Yellow Pensil Pea & Stylosanthes biflora \\
\hline
\end{tabular}




\begin{tabular}{|c|c|c|}
\hline Month & Common Name & Scientific Name \\
\hline \multirow[t]{6}{*}{ August } & Mexican Clover & Richardia spp. \\
\hline & Palm & many species \\
\hline & Primrose Willow & Ludwigia spp. \\
\hline & Shrubby False Buttonweed & Spermacoce verticillata \\
\hline & Smart Weed & Polygonum spp. \\
\hline & Spanish Needle & Bidens spp. \\
\hline \multirow[t]{6}{*}{ September } & Mexican Clover & Richardia spp. \\
\hline & Palm & many species \\
\hline & Primrose Willow & Ludwigia spp. \\
\hline & Shrubby False Buttonweed & Spermacoce verticillata \\
\hline & Smart Weed & Polygonum spp. \\
\hline & Spanish Needle & Bidens spp. \\
\hline \multirow[t]{7}{*}{ October } & Marlberry & Ardisia escallonioides \\
\hline & Mexican Clover & Richardia spp. \\
\hline & Primrose Willow & Ludwigia spp. \\
\hline & Shrubby False Buttonweed & Spermacoce verticillata \\
\hline & Smart Weed & Polygonum spp. \\
\hline & Spanish Needle & Bidens spp. \\
\hline & White Vine & Sarcostemma clausum \\
\hline \multirow[t]{5}{*}{ November } & Mexican Clover & Richardia spp. \\
\hline & Powderpuff Tree & Calliandra haematocephala \\
\hline & Primrose Willow & Ludwigia spp. \\
\hline & Shrubby False Buttonweed & Spermacoce verticillata \\
\hline & Spanish Needle & Bidens spp. \\
\hline \multirow[t]{5}{*}{ December } & Maple & Acer spp. \\
\hline & Mexican Clover & Richardia spp. \\
\hline & Primrose Willow & Ludwigia spp. \\
\hline & Spanish Needle & Bidens spp. \\
\hline & Wild Radish/Wild Mustard & Raphanus raphanistrum \\
\hline
\end{tabular}

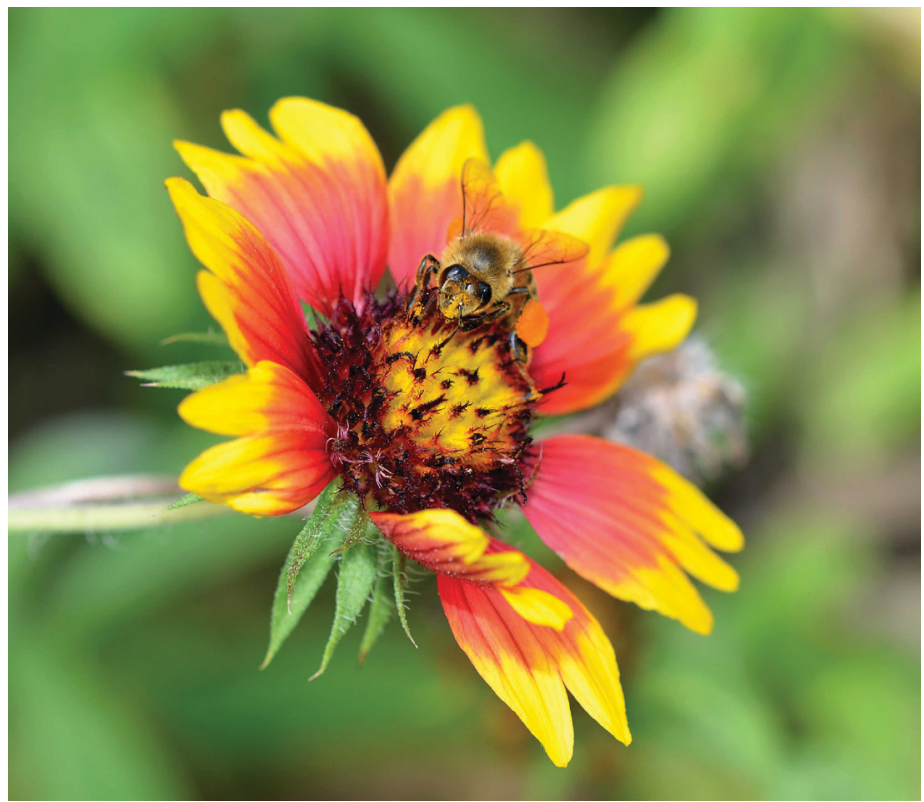

Figure 4. Honey bee on Indian blanket flower. 